\title{
OPTICAL AND STRUCTURAL CHARACTERISTICS OF YAG: Eu3+, Tb3+ ,Ce3+ LASER SINTERED CERAMICS
}

\author{
dos Santos, J. C. A.*; da Silva, E. P.; da Silva, R. S.
}

Physics Department, Federal University of Sergipe - UFS, Brazil

*jerrecristiano@gmail.com

\begin{abstract}
Yttrium aluminum garnet $\left(\mathrm{Y}_{3} \mathrm{Al}_{5} \mathrm{O}_{12}\right.$ or $\left.\mathrm{YAG}\right)$ has received much attention because of its interesting optical and mechanical properties. When doped with rare earth elements, it becomes a luminescent material with a wide variety of applications such as host material for solid-state laser, white light emitting diode (WLEDs) and scintillator material. Recently, a new method of sintering materials using a $\mathrm{CO}_{2}$ laser as main heating source has been employed. This method has possibilited to obtain ceramics with different physics properties when compared to conventional sintered samples. In this work, YAG: $\mathrm{Ce}^{3+}, \mathrm{Tb}^{3+}, \mathrm{Eu}^{3+}$ powders were synthesized by the polymeric precursor method and sintered by laser sintering technique. The main advantages of this sintering method are the rapid processing, the very high heating and cooling rates, and the possibility of sintering materials with high melting point. The ceramics characterization was done by X-ray diffraction, thermal analysis, scanning electronic microscopy, radioluminescence and photoluminescence techniques. The results showed that the laser sintering process was effective for obtaining high relative density YAG: $\mathrm{Ce}^{3+}, \mathrm{Tb}^{3+}, \mathrm{Eu}^{3+}$ ceramics with homogeneous grain size distribution. The radioluminescence and photoluminescence spectrum of the samples presented the characteristic emission of dopant elements. The structural characterizations were performed using the Rietveld method in all samples.
\end{abstract}

